\title{
Some Details of Statistical Mechanics of Many-Body Systems in the Presence of a Measurable Minimal Length
}

\author{
A. AlizadeH ${ }^{a, *}$ AND K. NOZARI ${ }^{b, c}$ \\ ${ }^{a}$ Department of Physics, Islamic Azad University, Sari Branch, Sari, Iran \\ ${ }^{b}$ Department of Physics, University of Mazandaran, P.O. Box 47416-95447, Babolsar, Iran \\ ${ }^{c}$ Center for Excellence in Astronomy and Astrophysics (CEAA-RIAAM), P.O. Box 55134-441, Maragha, Iran
}

(Received 5 November 2016; in final form 25 July, 2017)

\begin{abstract}
Different approaches to quantum gravity proposal such as string theory, doubly special relativity, and also black holes physics, all commonly address the existence of a minimal measurable length of the order of the Planck length. One way to apply the minimal length is changing the Heisenberg algebra in the phase space which is known as the generalized uncertainty principle. It is essential to apply this feature on the statistical mechanics of many body systems in the presence of a measurable minimal length scale in order to see the roles of this natural cutoff on physical phenomena. In this paper, some details of statistical mechanics of many body systems that have not been studied carefully in literature are studied in the presence of minimal length scale. The issues such as isomerization, the Liouville theorem, virial theorem and equipartition theorem are studied in this setup with details and the results are explained thoroughly.
\end{abstract}

DOI: 10.12693/APhysPolA.132.1329

PACS/topics: quantum gravity phenomenology, statistical physics, many-body systems, minimal measurable length

\section{Introduction}

A common feature of all existing approaches to quantum gravity is the existence of a minimal measurable length of the order of the Planck length [1-10]. This minimal length scale imposes limitation on the complete resolution of spacetime adjacent points in high energy regime and gives a fuzzy structure to spacetime manifold. A direct consequence of this minimal measurable length is discreteness of space at quantum gravity level. An interesting property of a minimal measurable length is correcting the relations between position and momentum operators in ordinary quantum mechanics, the result of which is called the generalized (gravitational) uncertainty principle (GUP) [11].

The simplest form of such relations can be written in one-dimensional form as

$$
\Delta x \Delta p \geq \frac{\hbar}{2}\left(1+\beta(\Delta p)^{2}+\gamma\right)
$$

where $\beta$ and $\gamma$ are positive quantities which are independent of $\Delta x$ and $\Delta p$, but may in general depend on the expectation values $\langle x\rangle$ and $\langle p\rangle$. The usual Heisenberg uncertainty relation can be recovered in the limit of $\beta=\gamma=0$. It is easy to see that this relation implies a minimal position uncertainty as $(\Delta x)_{\min }=\hbar \sqrt{ } \beta$. As a result, there is a minimal measurable length scale in measurement of position of a test particle and also probing spacetime structure. For a more general discussion on such a deformed Heisenberg algebras, especially in three dimensions, see $[8,12]$. Now, it is possible to realize Eq. (1) from the following relation between position

*corresponding author; e-mail: adele.alizadeh56@gmail.com and momentum operators:

$$
X=x, \quad P=p\left(1+\beta p^{2}\right),
$$
where $p^{2}=p_{i} p_{i}$ and we take $\gamma=\beta\langle p\rangle^{2}$. So we find

$$
\left.\hat{x}_{i}, \hat{p}_{j}\right]=\mathrm{i} \hbar\left(1+\beta p^{2}\right) \delta_{i j}, \quad\left[\hat{p}_{i}, \hat{p}_{j}\right]=0,
$$

where a hat marks operator character of the quantity. The commutation relations for the coordinates are obtained as

$$
\left[\hat{x}_{i}, \hat{x}_{j}\right]=2 \mathrm{i} \hbar \beta\left(\hat{p}_{i} \hat{x}_{j}-\hat{x}_{i} \hat{p}_{j}\right),
$$

which means that in more than one dimension, GUP naturally implies a non-commutative geometric generalization of the ordinary position space. In a statistical mechanics point of view, the microstates of a given classical system may be defined by $3 N$ position coordinates $x_{1}, \ldots, x_{3 N}$ and $3 N$ momenta $p_{1}, \ldots, p_{3 N}$, where $N$ is the number of particles in the system. In a geometric picture, the set of coordinates $\left(x_{i} p_{j}\right)$, where $i=1, \ldots, 3 N$, may be considered as a point in a $6 N$-dimensional space, the so-called phase space of the system. Since the coordinates $x_{i}$ and $p_{i}$ are varying with time, the dynamics of the whole system can be determined by using the Hamiltonian equations of motion for each of these coordinates as follows:

$$
\begin{aligned}
& \dot{x}_{i}=\left\{x_{i}, H\right\}=\left\{x_{i}, p_{j}\right\} \frac{\partial H}{\partial p_{i}}+\left\{x_{i}, x_{j}\right\} \frac{\partial H}{\partial x_{j}}, \\
& \dot{p}_{i}=\left\{p_{i}, H\right\}=-\left\{x_{j}, p_{i}\right\} \frac{\partial H}{\partial x_{j}},
\end{aligned}
$$

where $H\left(x_{i}, p_{j}\right)$ is the Hamiltonian of the system. We note that we have not included the Poisson bracket of $p_{i}$ and $p_{j}$ since we assume that the modified momentum still commutes.

Over the past few years, a number of research works have been devoted to the area of statistical mechanics in the GUP framework [13]. For instance, the ther- 
modynamics of the ideal gas and ultra-relativistic gas in micro-canonical ensemble in the GUP framework are studied in [14]. For harmonic oscillators and ideal gases in canonical ensembles with GUP see [15]. The deformed density matrix is studied in [16] and modified uncertainty relations for inverse temperature and internal energy are addressed in [17]. Black body radiation with minimal length effects is considered in [18]. As usual, the microstates of any physical system are determined by quantum mechanics and the corresponding energy levels should be obtained from the Schrödinger equation [19]. In the GUP framework, the Schrödinger equation becomes a non-linear or higher order differential equation and it is not easy to solve it analytically in general. For example, for the wave function and energy spectrum of harmonic oscillator see [20] and [21]. A particular non-linear Schrödinger equation in GUP framework is proposed in $[22,23]$. For the higher order modified Schrödinger equation for quantum mechanical systems, see [24]. In the GUP framework, the commutation relations take the following forms:

$$
\begin{aligned}
& \left\{x_{i}, x_{j}\right\}=2 \beta\left(p_{i} x_{j}-p_{j} x_{i}\right), \\
& \left\{x_{i}, p_{j}\right\}=\left(1+\beta p^{2}\right) \delta_{i j}, \quad\left\{p_{i}, p_{j}\right\}=0 .
\end{aligned}
$$

According to the Darboux theorem [25], it is always possible to find canonically conjugate variables $x_{i}(x, p)$ and $p_{i}(x, p)$ such that they satisfy the commutation relations (6). With these preliminaries, now we are in a position that we can focus on some statistical issues related to many-body systems in the presence of a natural cutoff as a minimal measurable length encoded in GUP.

\section{Isomerization theorem with minimal length}

We restrict our attention to equations just up to the first order in GUP parameter, $\beta$. First of all, we consider the expectation value of the quantity $x_{i} \boldsymbol{\nabla}\left(1-\beta \nabla^{2}\right) \cdot \boldsymbol{H}$ in the presence of the minimal measurable length in GUP. Note that we prefer to compute the statistical average of this quantity since this average is more conclusive on statistical ground than the usual form of this operator. In which follows, $H(q, p)$ is the Hamiltonian of the system where $x_{i}$ and $x_{j}$ are formally each of the $6 N$ generalized phase space coordinates as $(q, p)$. In our canonical ensemble and up to first order in $\beta$ we have

$$
\begin{gathered}
\left\langle x_{i} \boldsymbol{\nabla}\left(1-\beta \nabla^{2}\right) \cdot \boldsymbol{H}\right\rangle=\left\langle x_{i} \frac{\partial H}{\partial x_{j}}-\beta x_{i} \frac{\partial^{3} H}{\partial x_{j}^{3}}\right\rangle= \\
\int\left(x_{i} \frac{\partial H}{\partial x_{j}}-\beta x_{i} \frac{\partial^{3} H}{\partial x_{j}^{3}}\right) \mathrm{e}^{-\gamma H} \mathrm{~d} w / \int \mathrm{e}^{-\gamma H} \mathrm{~d} w .
\end{gathered}
$$

The integral in the numerator can be calculated by integration on $x_{j}$ to find

$$
\begin{gathered}
\int\left(x_{i} \frac{\partial H}{\partial x_{j}}-\beta x_{i} \frac{\partial^{3} H}{\partial x_{j}^{3}} \mathrm{e}^{-\gamma H}\right) \mathrm{d} w= \\
\int\left(\frac{1}{\gamma}-\beta \frac{1}{\gamma^{3}}\right) \delta_{i j} \mathrm{e}^{-H \gamma} \mathrm{d} w .
\end{gathered}
$$

The coefficient $\frac{\partial x_{i}}{\partial x_{j}}$ in the remaining integral equals with $\delta_{i j}$ comes out of the integral and we find

$$
\begin{aligned}
& \left\langle x_{i} \frac{\partial H}{\partial x_{j}}-\beta x_{i} \frac{\partial^{3} H}{\partial x_{j}^{3}}\right\rangle= \\
& \quad \int\left(x_{i} \frac{\partial H}{\partial x_{j}}-\beta x_{i} \frac{\partial^{3} H}{\partial x_{j}^{3}}\right) \mathrm{e}^{-\gamma H} \mathrm{~d} w / \int \mathrm{e}^{-\gamma H} \mathrm{~d} w= \\
& \quad \int\left(\frac{1}{\gamma} \delta_{i j}-\beta \frac{1}{\gamma^{3}} \delta_{i j}\right) \mathrm{e}^{-\gamma H} \mathrm{~d} w / \int \mathrm{e}^{-\gamma H} \mathrm{~d} w= \\
& \left(\frac{1}{\gamma} \delta_{i j}-\beta \frac{1}{\gamma^{3}} \delta_{i j}\right) .
\end{aligned}
$$

Therefore we find

$$
\begin{gathered}
\left\langle x_{i} \frac{\partial H}{\partial x_{j}}-\beta x_{i} \frac{\partial^{3} H}{\partial x_{j}^{3}}\right\rangle=\left(\frac{1}{\gamma}-\beta \frac{1}{\gamma^{3}}\right) \delta_{i j}= \\
{\left[k T\left(1-\beta(k T)^{2}\right)\right] \delta_{i j} .}
\end{gathered}
$$

The second term in the right hand side with coefficient $\beta$ is a term that has been emerged in the presence of the minimal measurable length encoded in GUP. Note that the standard result can be recovered easily in the limit of $\beta \rightarrow 0$. In the particular case with $x_{i}=x_{j}=p_{i}$, Eq. (7) turns out to the following form:

$$
\left\langle p_{i} \frac{\partial H}{\partial p_{j}}-\beta p_{i} \frac{\partial^{3} H}{\partial p_{j}^{3}}\right\rangle=k T\left(1-\beta(k T)^{2}\right)
$$

While the equation for $x_{i}=x_{j}=q_{i}$ will be as follows:

$$
\left\langle q_{i} \frac{\partial H}{\partial q_{j}}-\beta q_{i} \frac{\partial^{3} H}{\partial q_{j}^{3}}\right\rangle=k T\left(1-\beta(k T)^{2}\right) .
$$

Summing over all $i$, from $i=1, \ldots, 3 N$, we get

$$
\begin{aligned}
& \sum_{i=1}^{3 N}\left\langle p_{i} \frac{\partial H}{\partial p_{j}}-\beta p_{i} \frac{\partial^{3} H}{\partial p_{j}^{3}}\right\rangle=3 N k T\left(1-\beta(k T)^{2}\right), \\
& \sum_{i=1}^{3 N}\left\langle q_{i} \frac{\partial H}{\partial q_{j}}-\beta q_{i} \frac{\partial^{3} H}{\partial q_{j}^{3}}\right\rangle=3 N k T\left(1-\beta(k T)^{2}\right) .
\end{aligned}
$$

In several interesting physical problems, the Hamiltonian is a quadratic function of the coordinates. So, it can be written by a canonical transformation as follows:

$$
H=\sum_{j} A_{j} p_{j}^{2}+\sum_{j} B_{j} Q_{j}^{2},
$$

where $p_{j}$ and $Q_{j}$ are conjugate canonical variables while $A_{j}$ and $B_{j}$ are specific constants of the problem. For such a system, we obviously have

$$
\sum_{j}\left(p_{j} \frac{\partial H}{\partial p_{j}}-\beta p_{j} \frac{\partial^{3} H}{\partial p_{j}^{3}}+q_{j} \frac{\partial H}{\partial q_{j}}-\beta q_{j} \frac{\partial^{3} H}{\partial q_{j}^{3}}\right)=2 H
$$

where $\mathrm{H}$ is the GUP-deformed Hamiltonian of the system. Regarding the Eqs. (8) and (9) we get

$$
\langle H\rangle=\frac{1}{2} f k T\left[1-\beta(k T)^{2}\right],
$$

where $f$ is the number of non-zero coefficients. There- 
fore, it can be concluded that the GUP-deformation of the generalized Hamiltonian via the expression $\frac{1}{2} k T\left[1-\beta(k T)^{2}\right]$ has a particular role in the internal energy of the statistical mechanical system and hence to the specific heat of the system. This is the isomerization theorem in the presence of the minimum measurable length scale.

We note that since we are working up to first order in $\beta$, then it is expected that our analysis is supplemented by a limiting temperature at which these results are reliable. In fact the approximation $\beta(k T)^{2} \ll 1$ is a good assumption in high energy regime governed by GUP. Without such an approximation, values such as the average energy given by (12) can become zero or even negative. The best current limit on beta gives a limit on temperature of the order of the Planck temperature, $10^{32} \mathrm{~K}$. We note also that in the doubly special relativity there is a maximal energy (momentum) for a test particle on the order of the Planck energy (Planck momentum) that results in accordingly a maximal temperature of the order of the Planck temperature.

\section{Virial theorem with minimal length}

The virial theorem states that, for a stable, selfgravitating, spherical distribution of equal mass objects (stars, galaxies, etc.), the total kinetic energy of the objects is equal to minus $1 / 2$ times the total gravitational potential energy. In other words, the potential energy must be equal the kinetic energy with a factor of two.

In general, the expectation value of the sum of the products of the coordinates of the various particles in the system and the respective forces acting on them is referred to as the virial of the system. Using the relations (5) and (9), the following relation can be obtained:

$$
\begin{aligned}
& \sum_{i=1}^{3 N}\left\langle q_{i} \frac{\partial H}{\partial q_{j}}-\beta q_{i} \frac{\partial^{3} H}{\partial q_{j}^{3}}\right\rangle=3 N k T\left[1-\beta(k T)^{2}\right], \\
& \sum_{i=1}^{3 N}\left\langle q_{i} \frac{-\dot{p}}{\left\{q_{i}, p_{i}\right\}}-\beta q_{i} \frac{\partial^{2}}{\partial q_{i}^{2}} \frac{-\dot{p}}{\left\{q_{i}, p_{i}\right\}}\right\rangle= \\
& \quad 3 N k T\left[1-\beta(k T)^{2}\right], \\
& \sum_{i=1}^{3 N}\left\langle q_{i} \frac{-\dot{p}}{1+\beta p^{2}}-\beta q_{i} \frac{\partial^{2}}{\partial q_{i}^{2}} \frac{-\dot{p}}{1+\beta p^{2}}\right\rangle= \\
& \quad 3 N k T\left[1-\beta(k T)^{2}\right], \\
& -\sum_{i=1}^{3 N} \frac{1}{1+\beta p^{2}}\left\langle q_{i} \dot{p}-\beta q_{i} \frac{\partial^{2}}{\partial q_{i}^{2}}\left(q_{i} \dot{p}\right)\right\rangle= \\
& \quad 3 N k T\left(1-\beta(k T)^{2}\right) .
\end{aligned}
$$

Therefore we find

$$
v^{\{(G U P)\}}=-3 N k T\left[+\beta p^{2}-\beta(k T)^{2}\right] .
$$

This is the virial of a system in the presence of natural cutoff as a minimal measurable length. The terms containing $\beta$ are the corrections due to quantum gravity effect via existence of a minimal length scale. One recovers the standard result by putting $\beta=0$. This equation can be applied to a classical gas of non-interacting particles. In this case the only forces that acts on particles is the force which originates from the presence of the walls of the container. These forces can be exerted by an external pressure $P$, which is limited by the walls of the container. This, the so called $p d s$-force, depends on the element of the surface $\mathrm{d} s$, where the negative sign emerges since the force is inward while the normal to the container area is outward. In this case we have

$$
v_{0}=\left(\sum_{i} q_{i} F_{i}\right)_{0}=-P \oint_{s} \boldsymbol{r} \cdot \mathrm{d} \boldsymbol{s}=-P \oint_{V}(\boldsymbol{\nabla} \cdot \boldsymbol{r}) \mathrm{d} V
$$

In writing this relation the divergence theorem has been utilized. Considering the effects of quantum gravity vial the GUP, we find

$$
\begin{array}{r}
v^{\{G U P\}}=-P \int_{V} \nabla\left(1-\beta \nabla^{2}\right) \cdot \boldsymbol{r} \mathrm{d} V= \\
-P \int_{V}\left(1-\beta \nabla^{2}\right) \boldsymbol{\nabla} \cdot \boldsymbol{r} \mathrm{d} V=-3 P V .
\end{array}
$$

So we find

$$
v^{\{G U P\}}=-3 P V \text {. }
$$

Comparing (14) and (13), the following result is obtained up to the first order in $\beta$ :

$$
p V=N k T\left[1+\beta p^{2}-\beta(k T)^{2}\right] .
$$

This equation, which can be written as

$$
p=p(V, N, T)=\frac{N k T}{V}\left(1+\beta p^{2}-\beta(k T)^{2}\right)
$$

in order to resemble the Van der Waals form, is the equation of state of ideal gases in the phenomenological quantum gravity framework with a minimal length cutoff scale. The standard equation of state is recovered by setting $\beta=0$. The internal energy of the gas, with the theorem of isomerization (12), is given as follows:

$$
\langle H\rangle=\frac{3}{2} N k T\left(1-\beta(k T)^{2}\right) .
$$

$3 N$ is the number of degrees of freedom. As it is known, this energy is not the average kinetic energy of the system. Comparing this relation with relation (13) the following important result can be deduced:

$$
v^{\{G U P\}}=-\left(1+\beta p^{2}\right)\langle H\rangle .
$$

As usual, the $\beta$-dependent term has its origin in fact in the quantum gravity. Once again we note that we have performed our calculations up to the first order of the GUP parameter. It is important to emphasize also that the idea of minimal length has its origin in quantum gravity and here we have presented a toy model to see how this minimal length scale affects statistical mechanics of many-body systems at high temperature. At this point we note that the issue of composite system in a deformed space with minimal length has been studied by [26]. Following this seminal work, here we have studied some other statistical features of these composite systems. Naturally our results could be consistent with their results and this is actually the case. We refer also to [27] for another study on minimal length physics. 


\section{Summary and conclusions}

In this paper we have presented a toy model to study the effects of a minimal length scale on some important aspects of statistical mechanics of many-body systems. The origin of such a minimal length cutoff lies in quantum gravitational effect and is a common feature in all existing approaches to quantum gravity. Then, the issue of isomerization theorem is reconsidered in the presence of the minimal length cutoff. By calculating the expectation value of the Hamiltonian, we have shown that the internal energy of a statistical system changes in the presence of this natural cutoff. In this regard, since the internal energy and the specific heat $C_{V}$ are related quantities, we have shown that in the presence of the minimal measurable length the specific heat reduces due to the deformed algebra in the presence of the natural cutoff as the minimal length. This is related in essence to the reduction of the number of accessible microstates in the presence of the minimal length. This reduction is actually due to increment of the volume of the fundamental cell in phase space in comparison to the standard case.

We have studied also the issue of virial theorem in this setup. We have shown that this theorem gets modified considerably in this setup. In this manner, we have derived the modified equation of state of an ideal gas in the deformed space up to first order in the GUP parameter, $\beta$. The Liouville theorem is studied in the presence of natural cutoff as the minimal length and the deformation has been explained physically. We should stress here that this phenomenological implications that are shown themselves as correction terms in high energy (the Planck scale) regime, can be tested essentially in laboratory in future. These achievements provide direct test of quantum gravity but their detection needs very high energy accelerators. The counterparts of these effects in relativistic limit can be tested via ultra-high energy cosmic rays, too. In fact, existence of a natural cutoff as a minimal length modifies also the standard relativistic dispersion relation. It is possible to see the effect of this natural cutoff in ultra-high energy cosmic rays as a natural laboratory with energy on the scale of the Planck energy. In this respect, we refer the interested reader to the issue of GZK limit and also the Lorentz invariance violation in ultra-high energy cosmic rays experiments [28-30].

\section{Acknowledgments}

The work of K. Nozari has been supported financially by the Center for Excellence in Astronomy and Astrophysics of IRAN (CEAAI-RIAAM) under research Project No. 1/5411-1.

\section{References}

[1] G. Veneziano, Europhys. Lett. 199, 2 (1986).

[2] E. Witten, Phys. Today 24, 49 (1996).

[3] D. Amati, M. Ciafaloni, G. Veneziano, Phys. Lett. B 216, 41 (1989).

[4] D. Amati, M. Ciafaloni, G. Veneziano, Nucl. Phys. B 347, 550 (1990).

[5] D. Amati, M. Ciafaloni, G. Veneziano, Nucl. Phys. B 403, 707 (1993).
[6] K. Konishi, G. Paffuti, P. Provero, Phys. Lett. B 234, 276 (1990).

[7] L.J. Garay, Int. J. Mod. Phys. A 10, 145 (1995).

[8] M. Maggiore, Phys. Lett. B 319, 83 (1993).

[9] M. Maggiore, Phys. Lett. B 304, 65 (1993).

[10] F. Scardigli, Phys. Lett. B 452, 39 (1999).

[11] A. Kempf, G. Mangano, R.B. Mann, Phys. Rev. D 52, 1108 (1995)); A. Kempf, G. Mangano, Phys. Rev. D 55, 7909 (1997)); K. Nozari, A. Etemadi, Phys. Rev. D 85, 104029 (2012)); S. Ghosh, P. Roy, Phys. Lett. B 711, 423 (2012).

[12] M. Maggiore, Phys. Rev. D 49, 5182 (1994)); A. Kempf, J. Math. Phys. 35, 4483 (1994)); R.J. Adler, D.I. Santiago, Mod. Phys. Lett. A 14, 1371 (1999)); A. Kempf, Phys. Rev. D 63, 083514 (2001)); A. Kempf, J.C. Niemeyer, Phys. Rev. D 64, 103501 (2001).

[13] A. Alizadeh, J. Theor. Phys. 4, 14 (2015).

[14] K. Nozari, B. Fazlpour, Gen. Rel. Grav. 38, 1661 (2006)); K. Nozari, S.H. Mehdipour, Chaos Solitons Fractals 32, 1637 (2007).

[15] T. Fityo, Phys. Lett. A 372, 5872 (2008).

[16] A.E. Shalyt-Margolin, A.Ya. Tregubovich, arXiv:grqc/0207068; A.E. Shalyt-Margolin, J.G. Suarez, Int. J. Mod. Phys. D 12, 1265 (2003).

[17] A.E. Shalyt-Margolin, A.Ya. Tregubovich, arXiv:grqc/0204078.

[18] D. Mania, M. Maziashvili, Phys. Lett. B 705, 521 (2011).

[19] R.K. Pathria, P.D. Beale, Statistical Mechanics, Elsevier, 2011.

[20] X. Zhang, L. Shao, B.Q Ma, Astropart. Phys. 34, 840 (2011)); N. Chandra, S. Chatterjee, Phys. Rev. D 85, 045012 (2011).

[21] A. Lavagno, A.M. Scarfone, P.N. Swamy, Rep. Math. Phys. 55, 423 (2005)); A. Lavagno, P.N. Swamy, Found. Phys. 40, 814 (2010)); R.C. Venkatesan, A. Plastino, Physica A 389, 1159 (2010)); F.A. Brito, A.A. Marinho, Physica A 390, 2497 (2011)); A.A. Marinho, F.A. Brito, C. Chesman, Physica A 391, 3424 (2012).

[22] L.N. Chang, D. Minic, N. Okamura, T. Takeuchi, Phys. Rev. D 65, 125027 (2002)); K. Gemba, Z.T. Hlousek, Z. Papp, arXiv:0712.2078; Z. Lewis, T. Takeuchi, Phys. Rev. D 84, 105029 (2011).

[23] P. Pedram, Phys. Rev. D 85, 024016 (2012).

[24] P. Pedram, Int. J. Theor. Phys. 51, 1901 (2012).

[25] B. Vakili, M.A. Gorji, J. Statist. Mech. Theor. Exp. 2012, 10013 (2012); P. Pedram, K. Nozari, S.H. Taheri, J. High Energy Phys.1103, 093 (2011).

[26] C. Quesne, V.M. Tkachuk, Phys. Rev. A 81, 012106 (2010).

[27] C. Quesne, V.M. Tkachuk, SIGMA 3, 016 (2007).

[28] K. Nozari, S.D. Sadatian, Gen. Rel. Gravit. 40, 23 (2008).

[29] J. Alfaro, G. Palma, Phys. Rev. D 67, 083003 (2003).

[30] N.E. Mavromatos, Int. J. Mod. Phys. A 25, 5409 (2010) and references therein. 